Research Paper

\title{
Medaka Cleavage Embryos Are Capable of Generating ES-Like Cell Cultures
}

\section{Zhendong Li, Narayani Bhat", Dwarakanath Manali*, Danke Wang, Ni Hong, Meisheng Yi, Ruowen Ge, Yunhan Hong $\bowtie$}

Department of Biological Science, National University of Singapore, 10 Kent Ridge Crescent, Singapore 119260, Singapore

* This work contains part of thesis of these authors.

$\triangle$ Corresponding author: Prof. Yunhan Hong, Department of Biological Sciences, National University of Singapore, 10 Kent Ridge Crescent, Singapore 119260. Fax: +65 6779 2486; Tel: +65 6516 2915; Email: dbshyh@nus.edu.sg

(C) Ivyspring International Publisher. This is an open-access article distributed under the terms of the Creative Commons License (http://creativecommons.org/ licenses/by-nc-nd/3.0/). Reproduction is permitted for personal, noncommercial use, provided that the article is in whole, unmodified, and properly cited.

Received: 2011.01.01; Accepted: 2011.04.01; Published: 2011.04.15

\begin{abstract}
Mammalian embryos at the blastocyst stage have three major lineages, which in culture can give rise to embryonic stem (ES) cells from the inner cell mass or epiblast, trophoblast stem cells from the trophectoderm, and primitive endoderm stem cells. None of these stem cells is totipotent, because they show gene expression profiles characteristic of their sources and usually contribute only to the lineages of their origins in chimeric embryos. It is unknown whether embryos prior to the blastocyst stage can be cultivated towards totipotent stem cell cultures. Medaka is an excellent model for stem cell research. This laboratory fish has generated diploid and even haploid ES cells from the midblastula embryo with $\sim 2000$ cells. Here we report in medaka that dispersed cells from earlier embryos can survive, proliferate and attach in culture. We show that even 32-cells embryos can be dissociated into individual cells capable of producing continuously growing ES-like cultures. Our data point to the possibility to derive stable cell culture from cleavage embryos in this organism.
\end{abstract}

Key words: cleavage, morula, blastula, stem cell culture, totipotency

\section{Introduction}

In mammals, fertilization results in a zygote, the totipotent stem cell that can give rise to all cell types of a developing embryo and adult. A pre-implantation mouse embryo at the blastocyst stage consists of three cell lineages, inner cell mass (ICM), trophectoderm (TE) and primitive endoderm (PE). TE is the first morphologically distinct cell type of the trophoblast lineage, which becomes discernible at the morula stage and gives rise to the placental trophoblasts. PE is the first morphologically distinct cell type of the extraembryonic endoderm, which becomes visible at the late blastocyst stage as a layer on the mural surface of the ICM and gives rise to the yolk sac endoderm. ICM forms the epiblast, the first morphologically distinct cell type of the fetal lineage. The epiblast gives rise to amnion, extraembryonic mesoderm and embryo proper [1].

The pre- and peri-implantation embryo in culture can produce stable cell lines that show characteristics of the three blastocyst lineages. The first prototypes of such cell lines consists of embryonic stem (ES) cells from the mouse ICM [2; 3], which closely resemble nascent epiblast [4]. ES cell lines have also been isolated from the epiblast in rat and mouse, which closely resemble the post-implantation epiblast and human ES cells in gene expression profiles and transcription factor networks [5; 6]. Trophoblast stem (TS) cell lines have been isolated from blastocysts in the mouse [7]. Extraembryonic endoderm (XEN) stem cell lines have been isolated from mouse blastocysts [8]. Thus, there are cell lines that represent the earliest stages of the fetal pathway in vitro and appear to be 
remarkably similar across mammalian species.

Although these stem cells are capable of self-renewal and differentiation, none of them is totipotent, because they originate from the committed blastocyst lineages and maintain the respective properties of their origins. For example, these mammalian stem cells exhibits gene expression profiles characteristic of their sources $[1 ; 7 ; 8]$. More importantly, upon blastocyst transplantation and morula aggregation, mammalian ES cells can contribute to the epiblast but not extraembryonic lineages [9], whereas TS and PS cells usually enter the TE and PE of extraembryonic lineages but not the epiblast and its derivatives in the embryo proper $[7 ; 8]$.

The blastocyst so far represents the earliest stage for cell culture derivation. The attempt towards totipotent stem cell cultures from earlier developing embryos has not been available. The use of blastocyst stage embryos has conceptually excluded the possibility for totipotent cell derivation by origin. Recently, induced pluripotent stem (iPS) cells have been obtained by forced expression of pluripotency transcription factors even in differentiated mammalian cells [10]. However, it is unclear whether this approach can lead to totipotent stem cell cultures. Recently, small molecules operating on several signaling pathways have been used to partially convert mouse epiblast stem cells to an earlier pluripotency state [11]. In addition, primary cultures from early embryos can produce a uniform population for transcriptome and/or proteome approaches to study early events such as early lineage restriction and cellular differentiation in a controllable conditions. For example, primary cultures of single zebrafish embryos at early stages has been used to study myogenesis [12].

We use medaka as a lower vertebrate model for stem cell biology. This laboratory fish is well-suited for analyzing vertebrate development [13]. In this organism, we have previously established a feeder-free culture system that allowed for derivation of diploid ES cells [14-16] from fertilization midblastula embryos as the equivalent of mammalian blastocysts, male germ stem cells from the adult testis [17] and even haploid ES cells from gynogenetic embryos[18; 19]. We are interested in the possibility towards totipotent stem cell derivation. As a first step, this study was aimed at determining the suitability of earlier embryos prior to the midblastula stage for cell isolation and culture. We show that single cells from medaka embryos ranging from the 32-cell early morula stage to the 1000-cell early blastula stage can survive and proliferate in culture on gelatin-coated multiwell plates. More importantly, the cells from these earlier embryos can form a monolayer during 6 days of culture and display phenotypes similar to ES cells. These data suggest the possibility for stem cell derivation from these early embryos in this organism.

\section{Materials and Methods}

Fish. Work with fish followed the guidelines on the care and use of animals for scientific purposes of the National Advisory Committee for Laboratory Animal Research in Singapore (permit number 27/09). Medaka was maintained under an artificial photoperiod of 14 -h light to $10-\mathrm{h}$ darkness at $26^{\circ} \mathrm{C}$ [20]. Embryogenesis was staged according to [21]. Medaka strains HB32C, SOK and $i^{1}$ were used for cell culture in this study. HB32C is a wild-type pigmentation strain from which diploid ES cell lines were derived[14-16], whereas $i^{1}$ is an albino strain from which haploid ES cells generated [18; 19]. SOK is one of the best permissive strains for ES cell culture [22].

Cell culture. Embryo manipulation, cell isolation and cultures were done essentially as described [14-16]. Briefly, fertilization embryos were treated with proteinase $\mathrm{K}(10 \mathrm{mg} / \mathrm{ml})$ for $60 \mathrm{~min}$ at $28^{\circ} \mathrm{C}$ to remove the attachment filaments, rinsed twice in phosphate-buffered saline (PBS) and sterilized in PBS- $0.1 \%$ bleach for $2 \mathrm{~min}$, and rinsed 5 times in PBS. Embryos were incubated in PBS and monitored for developmental stages under a stereo microscope at aseptic conditions. The chorion was manually torn with a pair of fine forceps through the yolk sac at the vegetal half. Cells were dissociated by gentle pipetting, rinsed 5 times by partial PBS changes and seeded into gelatin-coated 96-well plates containing $150 \mu \mathrm{l}$ of ESM2. Cell growth, attachment, proliferation and differentiation were monitored at regular intervals of culture at $28^{\circ} \mathrm{C}$ in air.

Microscopy and Photography. Observation and photography on Leica MZFIII stereo microscope, Zeiss Axiovertinvert 2 invert microscope and Axiovert 200 upright microscope with a Zeiss AxioCam M5Rc digital camera (Zeiss Corp., Germany) were as described previously [19].

\section{Results}

\section{Embryo stages and lineages in medaka}

In medaka, early embryos undergo rapid synchronous cell divisions until the midblastula stage with $\sim 2000$ cells. Medaka ES cells have previously been derived from the midblastula embryos. In this study, we chose 6 earlier stages to test their possibility for cell culture derivation. Four representative stages are illustrated in Fig. 1. In medaka, the early blastula stage has 1000 cells of three lineages, pluripotent deep cells that produce the future embryo proper, and two 
extraembryonic lineages called the envelop layer (EVL) and yolk syncytial layer (YSL) [21]. The deep cells, EVL and YSL are equivalent to the inner cell mass or epiblast, trophectoderm and primitive endoderm of the mouse blastocyst embryo [1]. Medaka morula embryos have 128, 256 and 512 cells, which form deep cells and EVL. Medaka cleavage embryos have 32 and 64 cells, which are indeterminate in lineage commitment and thus have a promise to generate totipotent stem cell cultures. These 6 stages thus appear to represent a hierarchy of restriction in totipotency/pluripotency.
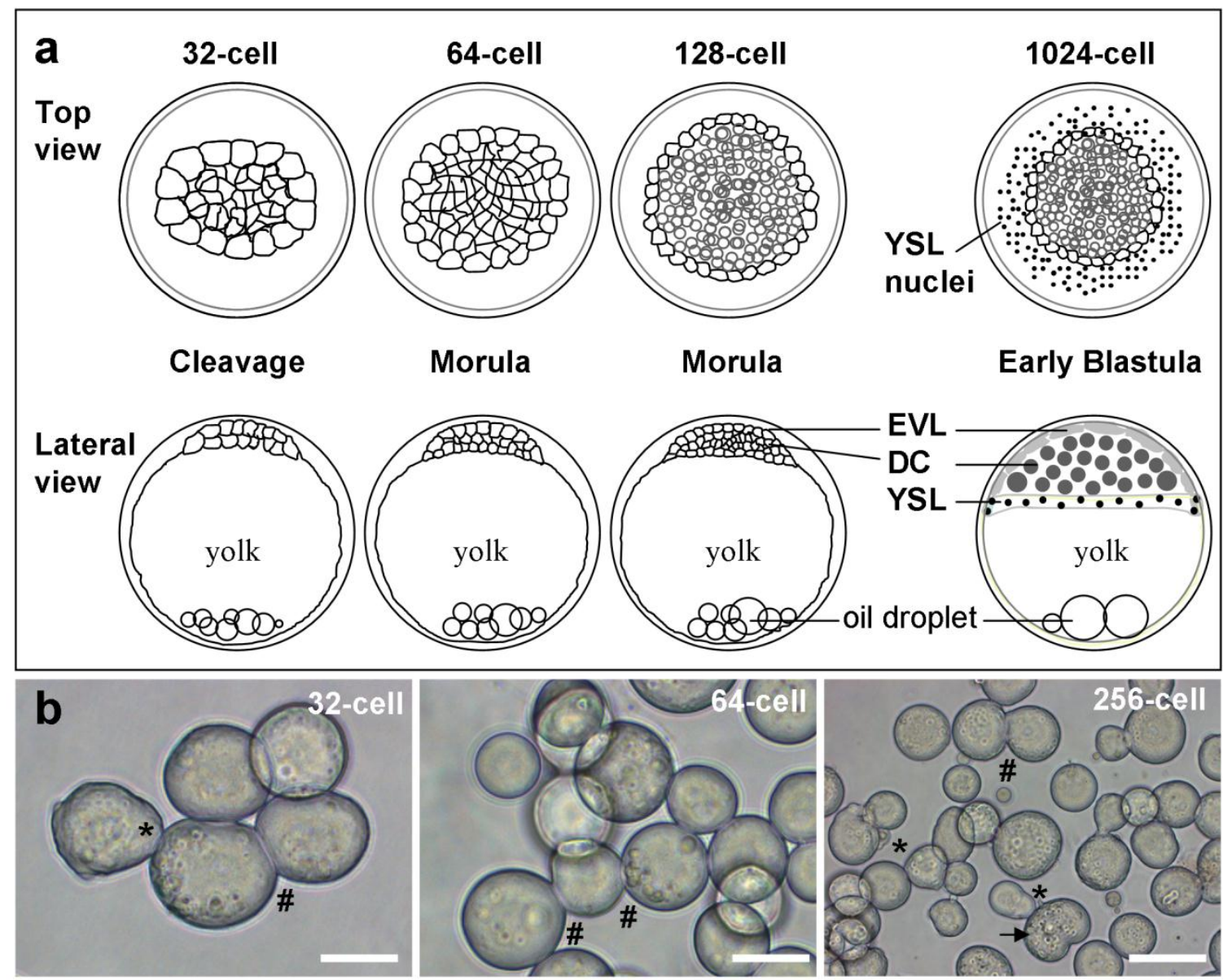

Fig. I. Medaka embryos and their cells. (a) Representative stages. EVL, envelope layer; DC, deep cells; YSL, yolk syncytial layer. (b) Isolated cells for culture. Developmental stages, pseudopodia (asterisks), intercellular bridges (\#) and dividing cells (arrow) are indicated. Scale bars, $50 \mu \mathrm{m}$.

\section{Cell isolation and culture}

We first tested various stages for the possibility and efficiency to isolated intact cells. We found that embryos from 128-cell stage onwards allowed for robust dissociation of individual cells, whereas cell isolation from 32- and 64-cell embryos was more demanding for skills. Upon isolation and seeding into culture plates, individual cells remained intact and viable as evidenced by pseudopodia (Fig. 1b). Isolated cells even from the same embryos exhibited varying sizes, and some of them were connected by intercellular bridges. Importantly, they continued active divisions and formed clusters of smaller daughter cells until $4 \mathrm{~h}$ of culture (Fig. 2), when cell death were apparent in certain cells without division in cultures from 64- and 128-cell embryos (Fig. 2a and b) and with one or two divisions in cultures from 256-cell embryos (Fig.2c). Dead cells appeared infrequently in cultures from 1024-cell embryos (Fig. 2d). Until one day of 
culture, cell attachment was seen for all the 6 stages of embryos (Fig. 3), as has been observed for cell culture initiation from diploid [14] and haploid midblastula embryos [19]. With regular medium changes, cell cultures from all the 6 stages survived and prolifer-
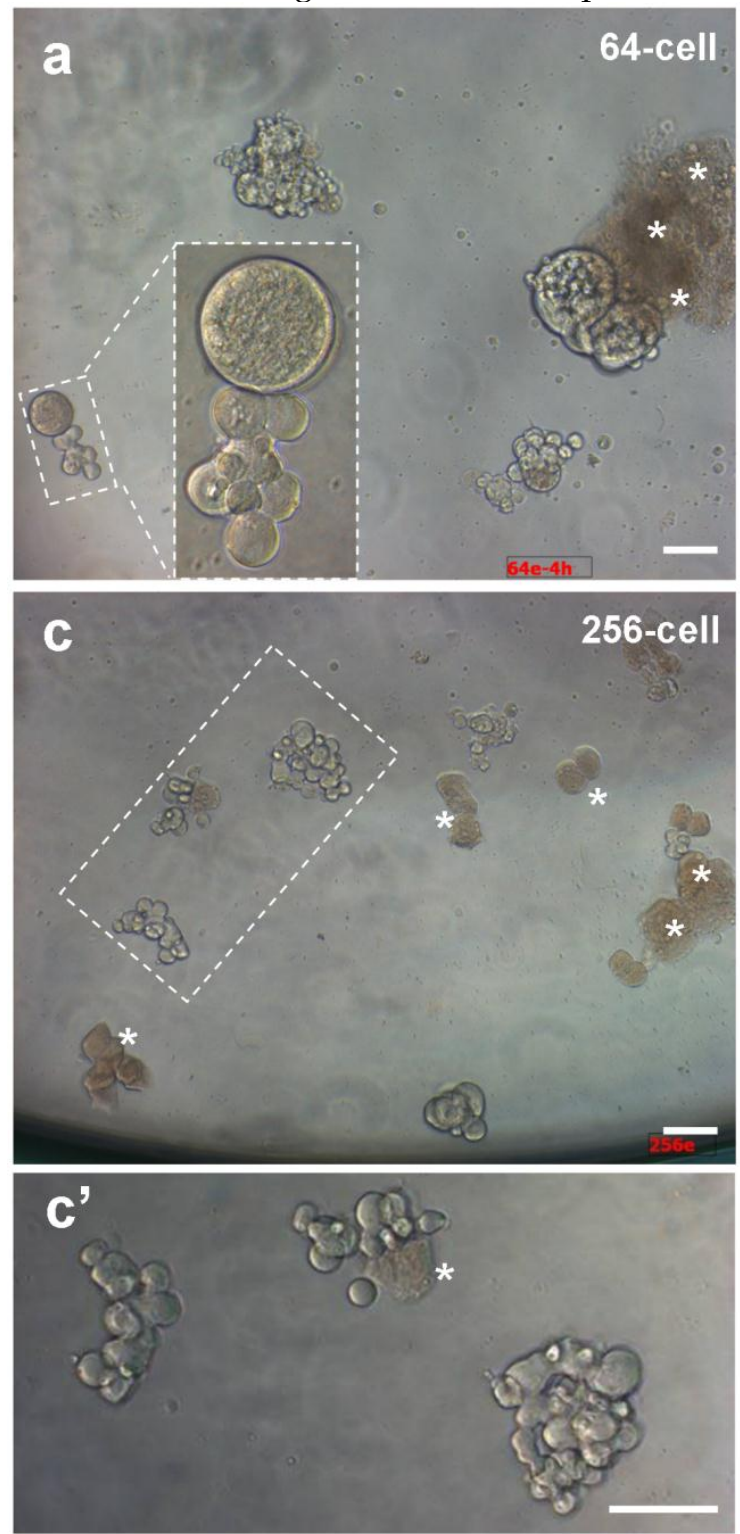

ated over the entire period of observation for up to 2 weeks. These data demonstrate the suitability of early medaka embryos prior to the midblastula stage for cell isolation and cell culture initiation.
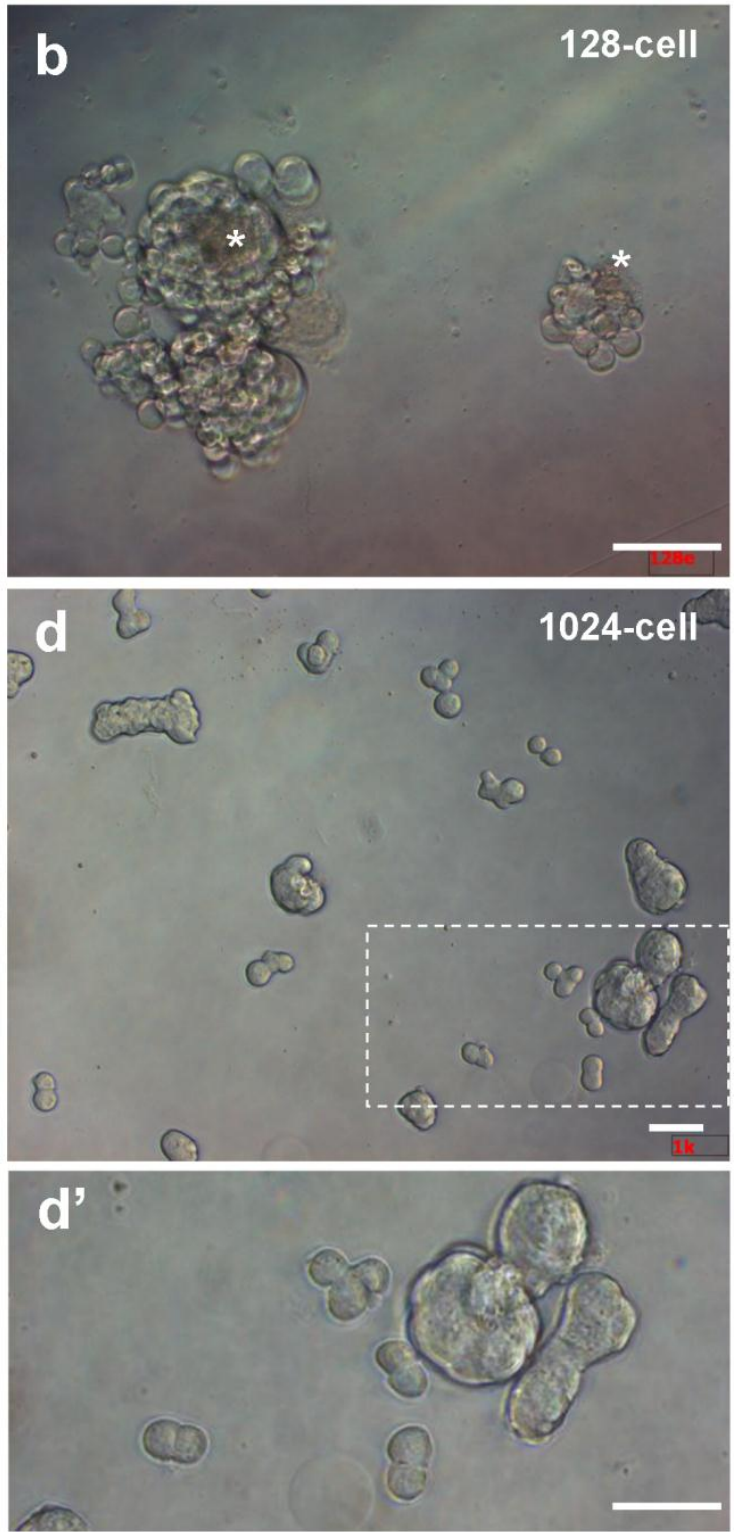

Fig. 2. Embryonic cells of different stages during 4 hours of culture. (a) 64-cell embryos. (b) I28-cell embryos. (c) 256-cell embryos. (d) 1024-cell embryos. (c' and d') Larger magnification views of framed areas in (c) and (d). Scale bars, 50 $\mu \mathrm{m}$.

\section{Phenotype of cell culture}

Like ES cell derivation from midblastula embryos (Hong and Schartl., 1996), cells from all the 6 stages continued active proliferation and at 6 days of culture, formed monolayers (Fig. 3a-f), which consisted of cells phenotypically resembling medaka ES cells [15; 19]. In addition, in cultures from 32-cell embryos, we observed YSL cells. These were giant cells with multiple nuclei at the periphery and typical syncytial cytoplasm at the center; and the cytoplasm exhibited motility and changed its shape by pseudopodial formation (Fig. 3g). YSL cells appeared also in cultures from embryos at other stages (Fig. 3h-l). Therefore, dissociated cells from embryos at 32- to 1024-cell stages can produce ES-like cell cultures and YSL cells, pointing to the possibility to derive pluripotent and even totipotent stem cell cultures from 
early medaka embryos.
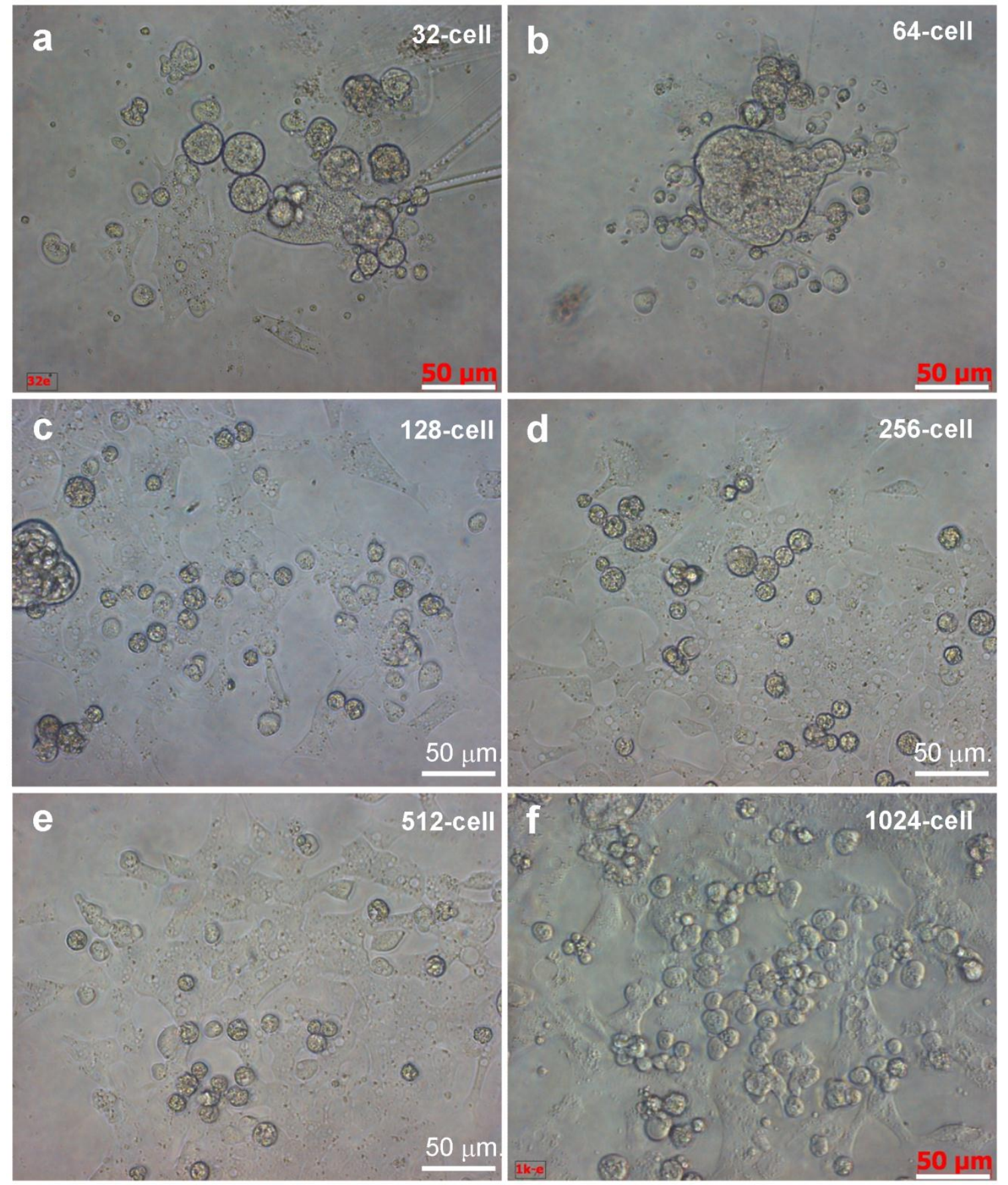

Fig. 3. Embryonic cells of different stages during 24 hours of culture. Scale bars, $50 \mu \mathrm{m}$. 

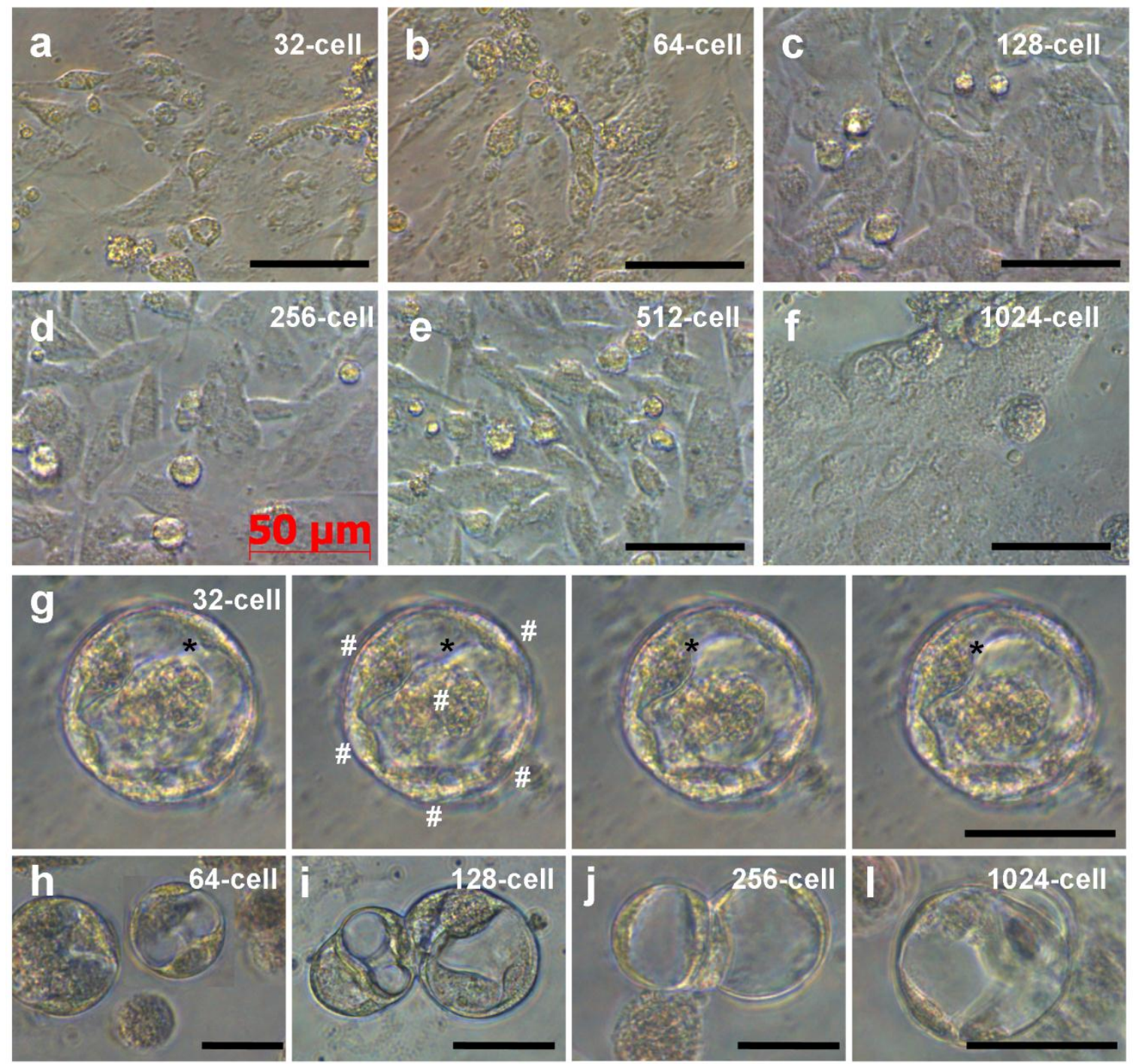

Fig. 4. Embryonic cells of different stages during 6 days of culture. (a-f) ES-like cells from embryos at indicated stages. (g) Serial micrographs of YSL cell, showing the motility of the cytoplasm (asterisks) residing in the center and multiple nuclei (\#) at the periphery. (h-l) YSL cells in cultures from embryos at stages indicated. Scale bars $\mu \mathrm{m}$.

\section{Discussion}

In this study we report the ability to isolate and culture early medaka embryonic cells as a step towards stem cell derivation from embryos before and after major lineage restriction. We show that medaka embryos as early as 32-cell stage have the accessibility for the isolation of viable cells capable of generating cell cultures. Importantly, embryonic cell cultures derived from earlier stages consist of cells exhibiting continuous growth and phenotypes that closely resemble well-characterized medaka ES cells derived from midblastula embryos $[14 ; 15 ; 19]$ suggesting that the competence for ES cell derivation is established at the 32-cell stage for stem cell derivation. In fish, no lineage restriction has been reported at this stage, which represents the earliest stage for cell culture derivation in vertebrates. In mammals, the blastocyst embryo after one or two events of lineage restriction leading to the inner cell mass, trophectoderm and primitive endoderm has successfully been used for cell culture, resulting in the derivation of ES cells [2; 23], trophoblasts stem cells [7] and extraembryonic endoderm stem cells [8]. It is unknown whether mammalian embryos at the 32-cell stage can produce cell cultures.

Interestingly, we have demonstrated that dispersed cells from embryos at all the 6 stages tested including the 32-cell stage can generate yolk syncytial layer cells of the primitive endoderm origin, one of 
the earliest extraembryonic lineages. It remains to be determined whether the YSL fate is predetermined at the 32-cell stage or specified under culture conditions. In medaka, the YSL originates from marginal blastomeres and its nuclei undergo endomitosis [21]. In zebrafish, YSL formation has been studied in more detail, where some marginal blastomeres are confluent with the yolk cell cytoplasm resulting from incomplete division collapse and deposit their nuclei and cytoplasm into the cytoplasmic cortex of the yolk cell, thereby forming the YSL, whose nuclei then undergo three to five rounds of endomitosis without cytokinesis [24]. The YSL is elusive for study due to difficulties in interfering specifically with YSL formation and morphogenesis [24]. Our finding that YSL cells can form and survive in cultures even from dissociated blastomeres of 32-cell embryos makes medaka an excellent model to study YSL formation in vitro.

Among the 6 tested stages, more advanced embryos are better in terms of ease and efficiency for cell isolation and culture. A larger cell size of earlier embryos is prone to mechanical damages during embryo manipulations, cell dissociation and seeding. For the possible derivation of totipotent stem cell cultures, however, the embryo even prior to the 32-cell stage is preferred. At the 32-cell stage, there are already two layers of cells that display differences in size and position, which may indicate the presence or onset of cell-cell interactions and different developmental pathways in differently positioned cells. Future work will determine whether 16- and even 8-cell embryos are amenable for cell isolation and culture.

In this study, our primary attention was on the possibility to isolate and cultivate early embryonic cells. We observed similar growth and proliferation during the entire 14-day period of culture between the 32-cell embryos obtained in this study and the midblastula embryos that eventually led to stable ES cell lines in our previous studies $[14 ; 15 ; 19 ; 25]$. Therefore, our results are not against the possibility that early embryonic cells can develop into stable cultures and even cell lines. In this regard, our detailed analyses of differential RNA expression of seven pluripotency genes and the telomerase reverse transcriptase in companion papers [26, 27] provide valuable information for characterizing putative stem cells at the molecular level.

\section{Acknowledgment}

We thank Jiaorong Deng for fish breeding, Veron Wong and Choy Mei Foong for laboratory management. This work was supported by the Biomedical Research Council of Singapore (R-08-1-21-19-585 \&
SBIC-SSCC C-002-2007).

\section{Conflict of Interests}

The authors have declared that no conflict of interest exists.

\section{References}

1. Rossant J. Stem cells and early lineage development. Cell. 2008; 132: 527-531.

2. Evans MJ, Kaufman MH. Establishment in culture of pluripotential cells from mouse embryos. Nature. 1981; 292: 154-156.

3. Martin GR. Isolation of a pluripotent cell line from early mouse embryos cultured in medium conditioned by teratocarcinoma stem cells. Proc Natl Acad Sci U S A. 1981; 78: 7634-7638.

4. Nichols J, Silva J, Roode M, Smith A. Suppression of erk signalling promotes ground state pluripotency in the mouse embryo. Development. 2009; 136: 3215-3222.

5. Brons IG, Smithers LE, Trotter MW, Rugg-Gunn P, et al. Derivation of pluripotent epiblast stem cells from mammalian embryos. Nature. 2007; 448: 191-195.

6. Tesar PJ, Chenoweth JG, Brook FA, Davies TJ, et al. New cell lines from mouse epiblast share defining features with human embryonic stem cells. Nature. 2007; 448: 196-199.

7. Tanaka S, Kunath T, Hadjantonakis AK, Nagy A, Rossant J. Promotion of trophoblast stem cell proliferation by fgf4. Science. 1998; 282: 2072-2075.

8. Debeb BG, Galat V, Epple-Farmer J, Iannaccone S, et al. Isolation of oct4-expressing extraembryonic endoderm precursor cell lines. PLoS One. 2009; 4: e7216.

9. Gertsenstein M, Nutter LM, Reid T, Pereira M, et al. Efficient generation of germ line transmitting chimeras from c57bl/6n es cells by aggregation with outbred host embryos. PLoS One. 2010; 5: e11260.

10. Takahashi K, Yamanaka S. Induction of pluripotent stem cells from mouse embryonic and adult fibroblast cultures by defined factors. Cell. 2006; 126: 663-676.

11. Zhou H, Li W, Zhu S, Joo JY, et al. Conversion of mouse epiblast stem cells to an earlier pluripotency state by small molecules. J Biol Chem 2010;285: 29676-29680.

12. Myhre JL, Pilgrim DB. Cellular differentiation in primary cell cultures from single zebrafish embryos as a model for the study of myogenesis. Zebrafish 2010;7: 255-266.

13. Wittbrodt J, Shima A, Schartl M. Medaka--a model organism from the far east. Nat Rev Genet. 2002; 3: 53-64.

14. Hong Y, Schartl M. Establishment and growth responses of early medakafish (Oryzias latipes) embryonic cells in feeder layer-free cultures. Molecular Marine Biol Biotechnol. 1996; 5: 93-104.

15. Hong Y, Winkler C, Schartl M. Pluripotency and differentiation of embryonic stem cell lines from the medakafish (oryzias latipes). Mech Dev. 1996; 60: 33-44.

16. Hong Y, Winkler C, Schartl M. Production of medakafish chimeras from a stable embryonic stem cell line. Proc Natl Acad Sci U S A. 1998; 95: 3679-3684.

17. Hong $\mathrm{Y}$, Liu T, Zhao H, Xu H, et al. Establishment of a normal medakafish spermatogonial cell line capable of sperm production in vitro. Proc Natl Acad Sci U S A. 2004; 101: 8011-8016.

18. Yi M, Hong N, Hong Y. Derivation and characterization of haploid embryonic stem cell cultures in medaka fish. Nat Protoc. 2010; 5: 1418-1430.

19. Yi M, Hong N, Hong Y. Generation of medaka fish haploid embryonic stem cells. Science. 2009; 326: 430-433. 
20. Li M, Hong N, Xu H, Yi M, et al. Medaka vasa is required for migration but not survival of primordial germ cells. Mech Dev. 2009; 126: 366-381.

21. Iwamatsu T. Stages of normal development in the medaka Oryzias latipes. Mech Dev. 2004; 121: 605-618.

22. Hong Y, Winkler C, Schartl M. Efficiency of cell culture derivation from blastula embryos and of chimera formation in the medaka (oryzias latipes) depends on donor genotype and passage number. Dev Genes Evol. 1998; 208: 595-602.

23. Thomson JA, Itskovitz-Eldor J, Shapiro SS, Waknitz MA, et al. Embryonic stem cell lines derived from human blastocysts. Science. 1998; 282: 1145-1147.

24. Carvalho L, Heisenberg CP. The yolk syncytial layer in early zebrafish development. Trends Cell Biol. 2010;20:586-592

25. Hong N, Li Z, Hong Y. Fish stem cell cultures. International Journal of Biological Sciences. 2011; 7(4): 392-402.

26. Wang D, Manali AD, Wang T, Bhat $\mathrm{N}$, et al. Identification of pluripotency genes in the fish medaka. International Journal of Biolological Sciences. 2011; 7(4): 440-451.

27. Rao F, Wang T, Li M, Li Z, et al. Medaka tert produces multiple variants with differential expression during differentiation in vitro and in vivo. International Journal of Biolological Sciences. 2011; 7(4): 426-439. 